\title{
Les routiers dans les films français : une image en mutation?
}

Highway truck drivers in French films: An evolving image

\section{Elisabeth Augustyn James}

\section{OpenEdition}

\section{Journals}

Édition électronique

URL : https://journals.openedition.org/rhcf/145

DOI : $10.4000 /$ rhcf. 145

\section{Éditeur}

Rails \& histoire

Édition imprimée

Date de publication : 1 décembre 2007

Pagination : 189-197

ISSN : 0996-9403

Référence électronique

Elisabeth Augustyn James, "Les routiers dans les films français : une image en mutation ? ", Revue d'histoire des chemins de fer [En ligne], 36-37 | 2007, mis en ligne le 10 mai 2011, consulté le 22 avril 2022. URL : http://journals.openedition.org/rhcf/145 ; DOI : https://doi.org/10.4000/rhcf.145 


\title{
Les routiers dans les films français : une image en mutation?
}

\author{
Elisabeth Augustyn James \\ Doctorante, université du Havre
}

Nous nous intéressons aux chauffeurs routiers de marchandises tels qu'ils apparaissent dans les films réalisés pour le cinéma et très souvent diffusés ensuite à la télévision française, ainsi qu'aux téléfilms réalisés pour cette même télévision. Notre étude se limite aux productions françaises. Nous évoquons toutefois les films dont l'action ne se passe pas en France, mais dont les héros sont des acteurs français. Nous ne prenons pas en compte en revanche les reportages sur le monde des routiers pour nous concentrer uniquement sur les fictions qui concernent les travailleurs de la route conduisant des poids lourds. Ceux-ci peuvent être des petits patrons, entrepreneurs de transport conduisant eux-mêmes, ou des salariés.

La question centrale est de savoir si le métier de routier a suscité des films qui servent à nourrir l'image dans le public se fait de ces hommes. Quelle est donc l'image du routier parmi le public ? Le routier apparaît souvent comme un homme un peu fruste, râleur mais courageux, qui peut devenir brutal si d'autres l'agacent ou le provoquent. Dans les différents films visionnés où il est question des camions, le héros peut être soit l'homme qui conduit le camion, soit la route, soit même, parfois, le véhicule lui-même. Pour rester fidèle au thème de notre recherche, nous nous intéressons donc à la première catégorie de films et téléfilms : celle dont le héros est un routier. Ils mettent en scène deux types de conducteurs : des routiers professionnels et des conducteurs occasionnels.

\section{Les films pour le cinéma}

\section{Les routiers et transporteurs professionnels}

Le film le plus connu impliquant des routiers est Le Salaire de la peur, d'Henri-Georges Clouzot (1953), un classique adapté du roman de Georges Arnaud, écrivain qui vécut en Amérique du Sud où il fut routier et chercheur d'or. Ce film, au suspense impressionnant, nous montre dans sa seconde partie le périlleux voyage qu'Yves Montand et Charles Vanel doivent entreprendre pour convoyer deux camions remplis 
de nitroglycérine. Ce voyage, s'il est mené à bien, leur offre la possibilité de retourner à Paris et de retrouver le métro dont Yves Montand conserve un ticket comme fétiche au fond de la poche de son bleu. Ce film est l'occasion d'une étude psychologique. Nous voyons ainsi le personnage interprété par Charles Vanel évoluer : de fier et fanfaron, il devient peu à peu nerveux, peureux, hésitant.

Cette fiction donne du chauffeur routier une image conforme à l'idée «classique » de l'époque : un homme viril, adroit, débrouillard, râleur, mais qui pense à ses collègues, qui prévoit leurs futures difficultés sur la route.

Dans Gas-oil (1955) de Gilles Grangier, sur un scénario de Michel Audiard, d'après le roman de George Bayle Du raisin dans le gasoil, avec Jean Gabin et Jeanne Moreau, l'action se situe dans la région de Clermont-Ferrand dans les années 1950. La vie d'un paisible routier, Jean Chape, est bouleversée par un accident. Il croit avoir écrasé un homme, un gangster notoire déjà mort, exécuté. Ses complices harcèlent Jean, pensant que celui ci a récupéré le produit d'un hold up sur le cadavre. Aidé de ses amis les routiers, Jean livre la bande à la police qui démasque l'assassin du gangster. Jean retrouve sa tranquillité et Alice, sa « fiancée », institutrice dans le Morvan, envoie sa démission au rectorat pour vivre dans l'ombre de son héros en région parisienne.

Le métier est bien décrit : Jean le routier, toujours en salopette, peut-être un peu trop propre, roule sans compter ses heures sur les routes, de jour, de nuit, comme les routiers des années 1950. C'est un homme "qui tient la chopine », aime la vie, le Beaujolais en quantité modérée. Il n'a pas peur des voyous qu'il combat seul d'abord, ensuite avec ses amis routiers. La tension générée par la conduite sur des routes difficiles et sinueuses est bien rendue. La solidarité professionnelle joue un rôle puisque c'est grâce à l'union des routiers que le "méchant » reçoit une juste punition.

La même année, Jean Gabin tourne Des gens sans importance, d'Henri Verneuil. Jean (Jean Gabin) est chauffeur de poids lourd, toujours en salopette. Un gars un peu bourru, un peu las de l'existence, mais robuste et courageux à sa manière. Il aime son métier. Il est marié. Il a trois enfants. Mais il ne s'entend plus avec sa femme, qui ne le comprend pas. Un jour, dans une auberge de routiers, tenue par Emile, ancien routier lui-même qui a perdu une jambe dans un accident du travail, Jean fait la connaissance d'une serveuse, Clotilde. L'amour les réunit. Elle tombe enceinte, «monte » à Paris et se fait avorter. Jean, cependant, a quitté sa famille et rejoint celle qu'il aime. Mais dans le 
camion qui pour la première fois les réunit, la jeune femme fait une hémorragie, perd connaissance tandis qu'ils se perdent dans le brouillard sur une petite route et que meuglent désespérément les veaux de la cargaison. Elle meurt à l'endroit même où ils se sont rencontrés.

Nous avons à faire ici à un mélodrame des plus sombres, tout est triste : les routes, le temps, la vie de famille. Seul Bertie interprété par Pierre Mondy, compagnon de route de Gabin et chauffeur, lui aussi, donne quelques touches de gaieté. Jean Gabin campe, encore une fois, un homme solide, toujours sur la route, ne comptant pas ses heures de travail. (Il roule $1500 \mathrm{~km}$ sans se reposer, passe la nuit du 24 décembre à rouler, dort deux heures cette même nuit dans un relais routier avant de repartir sur la route.) L'emploi d'un controlographe (rarissime pour l'époque) permet au chef de Gabin de savoir que ce dernier passe du temps dans le même relais routier. Lorsqu'il change son circuit, Gabin n'hésite pas, il frappe son chef et donne son compte. La technique permet ici d'espionner le travailleur, de mettre au jour ses fautes professionnelles et sa vie personnelle.

La déchéance de l'homme apparaît aussi dans le matériel qu'il conduit. Traditionnellement, dans le métier, les « grands routiers », qui parcourent de longues distances, sont mieux considérés, davantage respectés que les chauffeurs de bétaillère : le parallèle est clair entre la déchéance personnelle et professionnelle de Jean puisque de " grand routier », il devient, après une période sans travail, conducteur de bétaillère.

Un certain nombre de chauffeurs routiers, rencontrés lors d'entretiens, critiquent ce film, auquel ils reprochent de donner une vision caricaturale du routier cavaleur.

Avec Cent mille dollars au soleil (1963) Henri Verneuil signe un film d'aventures sur des dialogues de Michel Audiard, avec Jean-Paul Belmondo et Bernard Blier. Aux confins du Sahara, une entreprise de transports emploie comme chauffeurs des hommes venus de tous les coins d'Europe, des « durs » au passé souvent lourd. L'un d'eux disparaît avec un camion tout neuf. Le patron propose deux millions à un autre chauffeur s'il rattrape le fugitif et ramène le camion discrètement. Les routes sahariennes aux virages abrupts donnent l'occasion de prises de vue dépaysantes qui incitent le spectateur français à admirer la dextérité des conducteurs.

Là encore ce film n'est qu'un prétexte pour nous montrer un milieu de « têtes brûlées », de « durs », à l'attitude virile, vivant dans un cadre exotique : le Sahara. Le spectateur n'apprend rien sur le métier de routier. 
La Menace (1977), d'Alain Cornaud, appartient au genre policier et a pour toile de fond une entreprise de transport routier. Deux femmes s'affrontent pour un homme. La patronne d'Henri Savin, gérant de l'entreprise de transport routier, est retrouvée morte. Sa maitresse et lui sont soupçonnés.

L'essentiel du film réside dans la recherche du ou de la coupable du meurtre par la police. Un des rares chauffeurs-routiers français que nous croisons est un pauvre type qui sombre dans l'alcool. Henri Savin échappe à la police et commence à refaire sa vie au Canada. Il conduit un camion et monte un scénario qui laisse penser que sa disparition puis sa mort sont l'œuvre d'un gang qui rackette les routiers et tue ceux qui refusent de céder au chantage. Par un malheureux concours de circonstances, les routiers canadiens pensent avoir trouvé le chef de ce gang en la personne d'Henri Savin. Grâce à la Cibi, les routiers solidaires prennent Savin en chasse et broient la voiture qu'il conduit. C'est un des rares films où les routiers sont perçus négativement. Le meurtre se justifie aux yeux des routiers canadiens par l'inefficacité de la police. Comme dans Gas-oil, leur organisation, leur connivence, leur solidarité impressionnent. Il semble que rien ne puisse arrêter ces hommes et leur machine qui donne la mort (a fortiori celle d'un innocent).

Avec le film Le Grand Ruban (1990), drame réalisé par Philippe Roussel, nous accompagnons un routier, Jeff (Philippe Léotard), partant de Paris avec son chargement pour se rendre en Yougoslavie. Il rencontre au début de son parcours une fille "paumée », une prostituée de parking qu'il emmène avec lui. Il s'attache à elle et en tombe peu à peu amoureux.

Ce film, par certains aspects, se veut un documentaire réaliste sur le monde des routiers avec son quotidien difficile, les dessous de table que l'on donne aux douaniers pour obtenir un traitement plus rapide à la frontière, son vocabulaire spécifique...

Ce film transmet une image positive du routier : Jeff s'est « fait tout seul » car il a acheté son camion et il en est fier. En dehors de son travail, c'est un homme simple, il roule en motocyclette (c'est rare chez les routiers des années 1990) et habite dans une cité HLM ; il n’a pratiquement pas de défaut hormis sa spontanéité un peu brutale et virile. Il s'est même mis à vivre, pour l'aider moralement, avec la veuve d'un collègue routier mort sur la route. Toutes les femmes (la serveuse du restaurant, sa collègue et amie routière, la femme chez qui il loge en Yougoslavie), l'apprécient et disent de lui que c'est « un mec bien ». 
Dans ce film, comme dans les précédents, le spectateur assiste à une brève course-poursuite routière. Les bandits, rapidement mis hors d'état de nuire par Jeff le routier et son amie ne peuvent plus nuire aux travailleurs de la route.

\section{Les routiers occasionnels}

Le Camion blanc (1942) de Léo Joannon, avec des dialogues d'André Cayatte, raconte l'histoire d'un jeune mécanicien qui veut devenir chauffeur-routier. Il reçoit de Shabbas, chef des gitans du Nord, la mission de parcourir en sens inverse la route qu'a suivie tout au long de sa vie le roi des gitans défunt. Selon les rites gitans, la dépouille est placée dans un camion blanc et le périple de douze mille kilomètres à parcourir en quinze jours peut commencer. Des embûches surgissent sans cesse, suscitées par le conseiller des gitans du Sud, Courbassié. À l'issue de la randonnée, Shabbas victorieux est élu roi à son tour.

Ce film est plus la description amusante de l'itinéraire du camion à travers les paysages français qu'un film sur le métier de routier. L'épisode des éléphants dépassant le camion ensablé dans la baie du Mont saint-Michel reste un moment agréable.

L'Armoire volante (1948) de Carlo Rim met en scène Fernandel dans le rôle d'Alfred Puc qui espère hériter de sa tante acariâtre et avare, tante qui malgré son grand âge accompagne les déménageurs pour chercher ses meubles à la campagne. Une vague de froid s'étend et elle meurt en route. Les déménageurs déposent le cadavre dans une armoire et reviennent sur Paris. Leur camion est volé par des gangsters, alors qu'ils informent monsieur Puc de son deuil. Celui-ci entreprend un marathon, par monts et par vaux, chez les receleurs, dans un hôtel de passe, à la salle des ventes où il achète seize armoires du même modèle..., mais il ne peut retrouver ni armoire, ni tante. Un vrai cauchemar pour Fernandel.

Là encore, le film n'est qu'un prétexte pour apercevoir de manière plus ou moins loufoque des déménageurs, des camionneurs mais aussi des gens du milieu. Bref, ce n'est pas un film sur les routiers.

Impossible... pas français (1974) de Robert Lamoureux est un film adapté du roman policier de Horace Mac Coy: Un linceul n'a pas de poches. Il s'agit d'un film amusant, même « franchouillard", avec Pierre Mondy, Pierre Tornade et Jean Lefebvre, anciens chômeurs qui se transforment en camionneurs. Ils peuvent parvenir à la fortune s'ils arrivent à transporter un produit rare et coûteux de Barcelone au Havre. Après de multiples péripéties, ils arrivent enfin à leur destination. Aucune indication réelle n'est donnée sur le métier de routier. 
Nous devons mentionner également le film de Duras, Le Camion, tourné en 1977, avec Marguerite Duras et Gérard Depardieu, ensemble dans un salon, en train de discuter. Elle imagine un scénario de film où une femme aurait fait de l'auto-stop, un routier l'aurait fait monter dans son camion. Elle aurait beaucoup parlé d'elle tandis que le routier l'écouterait et resterait quasiment muet. Le film alterne des images de la lecture du scénario par Duras et Depardieu dans un salon et des images de camions à l'infini, d'intérieurs de camion, de camions bâchés en train de rouler...

Ce film renseigne sur la représentation que les gens peuvent se faire du routier : quelqu'un de serviable, mais peu bavard.

\section{Les téléfilms}

En 1963, quinze épisodes de treize minutes d'un feuilleton $\mathrm{La}$ Route, réalisé par Pierre Cardinal, sont diffusés sur Antenne 2. Ce feuilleton, classé dans la catégorie "aventures", nous raconte l'histoire de deux copains routiers, Dupuy et Mathieu, roulant à travers la France pour leur métier. Il semble que ce feuilleton ait laissé un bon souvenir chez les routiers car une campagne de signatures est actuellement engagée pour faire éditer cette œuvre en DVD.

Dans les années 1970, un autre feuilleton composé de vingtquatre épisodes de treize minutes, Le Pèlerinage, est diffusé chaque soir à $19 \mathrm{~h}$ 45. Il raconte les mésaventures de Raymond Colbi (Jean-Claude Bouillon), ex-mercenaire qui remet de l'ordre dans une scierie alsacienne lors d'un pèlerinage sur les lieux de son enfance. Cette scierie est menacée de rachat par un groupe. Ce feuilleton n'est pas centré sur les routiers, il permet juste d'entrevoir un véhicule grumier, utilisé pour les coupes de bois.

Vendredi 20 janvier 2006, un épisode de la série Boulevard du Palais était diffusé sur A2. Le titre de cet épisode était « Poids lourds». Un patron transporteur est assassiné sur son lieu de travail. Au début de l'enquête, la juge fait cette remarque : "Une société de transport est souvent la plaque tournante de trafics divers ", ce qui, dans le cas présent, s'avère exact. Un chauffeur fait le trafic de cigarettes et d'autres trichent avec leur chronotachygraphe. Même le patron «carotte les heures supplémentaires » de ses employés. Le peu de chauffeurs que le téléspectateur rencontre sont conformes à l'idée généralement admise des routiers : ils ont un code de l'honneur, se soutiennent entre eux, parlent peu et ne dénoncent pas les erreurs de leurs collègues. 


\section{Conclusion}

Les séries télévisées françaises à succès comme L'Homme $d u$ Picardie, Les Saintes Chéries ou La Route des années 1960 plaisaient au public, car il pouvait s'identifier aux personnages. Chacun y voyait son quotidien. Il semble que les téléfilms contemporains se passent désormais dans des couches sociales plus élevées de la population (médecins, vétérinaires, avocats...). Nous pouvons penser que l'image de l'ouvrier ou de l'artisan n'est pas vraiment positive ou du moins peu attractive. Dans les années 1950, le Français moyen éprouvait du plaisir à voir Gabin, vedette célèbre issue du peuple, se mettre dans la peau d'un homme simple comme lui, ouvrier. Il pouvait même s'identifier au héros. De nos jours, comme à la télévision, à part peut-être un cinéma réaliste et social anglais, attirant peu les foules, les héros des films ne sont quasiment jamais des ouvriers ou des artisans. Peut-on y voir un désir d'ascension sociale? Ou alors une envie d'oublier le quotidien?

Quant à l'image du routier - le «type » sympathique, un peu bougon, viril au grand cœur tel qu'il apparait dans le film mythique Le Salaire de la peur -, elle a évolué. Les derniers films véhiculent plutôt l'idée d'un homme qui mène une vie décalée, qui éprouve des difficultés à avoir une vie familiale et sociale dans les normes. Mais comme tous les héros, il a de grandes qualités.

Notons enfin que tous les films nous donnent une image d'un homme solitaire, jamais syndiqué, même s'il peut agir en concertation avec ses collègues.

\section{Films visionnés}

Le Camion blanc (1942), 1 h 52

Réalisateur: Léo Joannon; scénario : André-Paul Antoine, Léo Joannon ; dialoguiste : André Cayatte.

Avec François Périer (François Ledru), Jules Berry (Shabbas), Blanchette Brunoy (Germaine), Marguerite Moreno (la veuve du roi), Fernand Charpin (Courbassié).

L'Armoire volante (1948), 1 h 30

Réalisateur, scénario, adaptation, dialogues : Carlo Rim ; musique : Georges Van Parys.

Avec Fernandel (Alfred Puc), Pauline Carton (Madame Ovide), Antonin Berval (Grand Charles), André Bervil (Le premier habitué), Berthe Bovy (Madame Lea Lobligeois). 
Salaire de la peur (1952), 2 h 21

Réalisateur: Henri-Georges Clouzot; scénario: Henri-Georges Clouzot et René Wheeler, d'après le roman éponyme de Georges Arnaud ; musique: Georges Auric.

Avec Yves Montand (Mario), Charles Vanel (M. Jo), Peter van Eyck (Bimba), Antonio Centa (le chef du camp), Darling Légitimus (Miss Légitimus).

Gas-oil (1955), 1 h 32

Réalisateur: Gilles Grangier; scénario : Michel Audiard, Gilles Grangier, d'après une œuvre de Georges Bayle ; dialoguiste : Michel Audiard.

Avec Jean Gabin (Jean Chape ), Jeanne Moreau (Alice), Ginette Leclerc (Madame Scoppo), Camille Guérini (L'ancien), Albert Dinan (Emile Serin), Marcel Bozzuffi (Pierrot), Robert Dalban (Félix), Roger Hanin (Schwob), Gaby Basset (Camille Serin), Simone Berthier (Annie, une serveuse), Charles Bouillaud (Le gendarme dactylographe).

Des gens sans importance (1955), $1 \mathrm{~h} 41$

Réalisateur: Henri Verneuil, auteur de l'œuvre originale : Serge Groussard ; dialoguiste : François Boyer ; musique : Joseph Kosma.

Avec Jean Gabin (Jean Viard); Françoise Arnoul (Clotilde) ; Pierre Mondy (Berty) ; Yvette Etiévant (Solange) ; Paul Frankeur (Emile Barchandeau); Edmond Ardisson (un routier) ; Marcelle Arnold (la concierge); Jacques Ary (un routier).

100000 dollars au soleil (1963), $2 \mathrm{~h} 10$

Réalisateur : Henri Verneuil ; scénario : Marcel Jullian et Henri Verneuil, d'après le roman de Claude Veillot Nous n'irons pas an Nigéria; premier assistant réalisateur: Claude Pinoteau ; dialogues : Michel Audiard.

Avec Jean-Paul Belmondo (Rocco), Lino Ventura (Hervé Marec dit «le Plouc »), Bernard Blier (Mitch-Mitch), conduisant différents camions Berliet.

Impossible... pas français (1974), $1 \mathrm{~h} 30$

Réalisateur et scenario : Robert Lamoureux

Avec Jean Lefebvre, Pierre Mondy, Pierre Tornade, Robert Lamoureux, France Dougnac. 
Le Camion (1977)

Réalisateur, scénario : Marguerite Duras ; directeur de la photographie : Bruno Nuytten ; musique : Ludwig van Beethoven.

Avec Marguerite Duras (Elle), Gérard Depardieu (Lui).

La Menace (1977)

Réalisateur: Alain Corneau; scénario: Daniel Boulanger, Alain Corneau ; dialoguiste : Daniel Boulanger.

Avec Yves Montand (Henri Savin), Carole Laure (Julie Manet), Marie Dubois (Dominique Montlaur), Jacques Rispal (le chauffeur du poids lourd).

Le Long Ruban (1990). 